Katarzyna Kwapisz-Osadnik

Université de Silésie

\title{
LA PRÉPOSITION DE DANS UN CADRE COGNITIF
}

\section{The preposition de in a cognitive framework}

\begin{abstract}
A cognitive analysis of the uses of the preposition de in the contemporary French proposed in this article proves that 1 . the preposition de is a category containing several uses organized around prototypical uses; 2. there is a semantic invariant that makes possible distinguishing between the value of the preposition $d e$ and other prepositions that may appear in the same context (eg. a typewriter vs a writing machine); 3. simple and contracted forms are motivated by the frequency of usage based on preferential uses; 4 . the choice of the preposition is negotiated simultaneously on the conceptual, linguistic and discursive levels.
\end{abstract}

KEYWORDS: preposition, cognitive linguistics, prototypical uses, semantic invariant.

La préposition est une catégorie grammaticale qui, au niveau syntaxique, rend compte des rapports entre les autres constituants de la phrase. Beaucoup d'ouvrages lui sont consacrés (p.ex. Zielinski-Wibbelt 1993 ; Taylor 1993 ; Vandeloise 1993, 1995 ; Cervoni 1999 ; Przybylska 2002 ; Gilbert, Guimier, Krause 2009 ; Luraghi 2011 ; Giuliani 2013). Ces ouvrages représentent différentes approches : sémantique, fonctionnelle, pragmatique et cognitive. L'étude s'effectue soit à l'échelle macro (la préposition en tant que catégorie universelle), soit à l'échelle micro (la préposition comme ensemble des unités d'une langue donnée). Toutefois, on peut distinguer quelques tendances générales dégagées au terme de nombreuses analyses (Cervoni 1999) :

1. la préposition est une catégorie accessoire et redondante, c'est-à-dire qu'elle n'est pas toujours indispensable à la construction du sens (p.ex. Varsovie $10 \mathrm{~km})$;

2. le sens des prépositions est déterminé par un contexte précis (p.ex. arriver à l'école vs. arriver chez Marc);

3. étant éléments du système, les prépositions sont sémantiquement vides (p.ex. oublier de téléphoner);

4. la préposition est une catégorie syntaxique relationnelle, souvent dépourvue de sens (p.ex. une statue de Miron) ;

5. l'emploi des prépositions dépend des stratégies discursives et de l'intention communicationnelle (p.ex. au sujet de vs. à propos de) ;

6. l'emploi des prépositions se fonde sur les connaissances du monde (p.ex. on fait qqch. avec un instrument).

La présente étude se situe dans le cadre de la linguistique cognitive, et se réfère notamment aux notions de schéma sémantico-cognitif et d'invariant sémantique proposées par J.-P. Desclés (1997 et d'autres ouvrages ultérieurs). 
Le corpus est constitué surtout d'expressions où au lieu de la préposition de on peut en avoir d'autres, comme dans : une table de marbre vs. une table en marbre, une tarte de fraises vs. une tarte aux fraises, discuter la paix vs. discuter de la paix, continuer à faire qch. vs. continuer de faire qch.

Ce répertoire d'emplois de différentes prépositions dans les mêmes contextes sera le point de départ pour vérifier les hypothèses suivantes :

1. la préposition de est une catégorie qui s'organise autour des emplois prototypiques, qui relèvent de l'expérience directe du monde (Lakoff, Johnson 1988) et qui prend la forme d'un schéma (sémantico-cognitif);

2. tous les emplois de la préposition de sont compatibles avec la formule de son invariant sémantique ;

3. certains emplois ne sont pas sémantiquement motivés, mais ils relèvent de la fréquence d'usage.

Ceci dit, dans un premier temps, nous présenterons brièvement la vision cognitive de la catégorie de la préposition, pour passer ensuite à l'analyse du corpus. La dernière partie sera consacrée à une tentative de reconstruire le schéma sémantico-cognitif de la catégorie examinée et de vérifier la validité de la formule de l'invariant sémantique.

\section{LA PRÉPOSITION EN LINGUISTIQUE COGNITIVE}

Notre point de départ quant à la vision cognitive de la préposition est la conception de la grammaire proposée par R. Langacker (1987, 2008). Dans cette optique, on définit la préposition comme unité linguistique relationnelle. Les relations qu'elle exprime sont dépourvues de temporalité, de symétrie et de dynamique. Elles rendent compte de différentes façons de percevoir et de concevoir une même réalité à laquelle participent au moins deux objets, l'un appelé trajecteur, l'autre appelé landmark. Le trajecteur est l'objet du premier plan, par contre le landmark sert de point de référence par rapport auquel on saisit l'activité ou l'état du trajecteur. Comme toutes les unités de langue, la préposition a une structure de catégorie hiérarchiquement organisée à partir des emplois prototypiques et possédant un invariant sémantique. J.-P. Desclés et W. Banyś (1997 : 30) définissent l'invariant sémantique comme une formule « transcendant toutes les valeurs répertoriées d'une catégorie », et par conséquent, elle doit être intrinsèque à tous ses emplois. La notion d'invariant sémantique permet de saisir les différences de fonctionnement des catégories que l'on peut avoir dans les mêmes contextes d'expression, comme dans les exemples cités plus haut (une table de marbre / en marbre) ou dans d'autres (Je comprends qu'elle est / soit déçue).

En linguistique cognitive, il n'y a pas de catégories sémantiquement vides. Pour les prépositions, C. Vandeloise $(1993,1995)$ les définit comme ensemble de traits sémantiques qui sont de nature fonctionnelle et qui se configurent différemment selon le contexte dans lequel une préposition est employée. Ainsi, la préposition peut changer de sens d'un emploi à l'autre.

Le sens relève donc de l'emploi, c'est-à-dire qu'il est effet de l'imagerie. Cela veut dire que les catégories de langue, lexicales et grammaticales, au moment de percevoir un fragment de réalité, sortent de leur état de veille, activent leurs contenus conceptuels 
et deviennent porteuses de sens. Ainsi, les prépositions sont-elles aptes à exprimer divers rapports, par exemple un rapport spatial statique (sur la table), spatial directionnel (devant l'école), spatial dynamique (par le pont), temporel (en hiver), causal (mourir de faim), final (travailler pour vivre) et ainsi de suite.

Puisque les recherches cognitives en linguistique ont recours aux processus cognitifs de traitement des données, chaque analyse lexicale et grammaticale prend simultanément en compte le rôle des universaux perceptifs (p.ex. la préférence pour certaines directions ou pour le landmark, qui est un objet fixe) et des universaux linguistiques, ceux-ci prenant souvent la forme de figures plus ou moins schématiques.

\section{LA PRÉPOSITION DE : UN POINT DE VUE TRADITIONNEL}

La préposition de fonctionne sur 3 niveaux syntaxiques : dans un syntagme nominal (p.ex. une table de nuit, un roi de France, un fromage de chèvre, un vent d'orage, un coup de pied, la fille de mon collègue, une période de 3 mois, le mois de mars), dans un syntagme verbal (p.ex. sortir de l'école, venir de Paris, sauter de joie, servir de guide, se servir d'un pinceau, parler de, oublier de, rêver de) et dans un syntagme prépositionnel (p.ex. d'habitude, de rien, de bon matin, de bon cœur, de loin, de la part de, de mon côté, de plus, de travers, de toute façon).

Traditionnellement, dans une phrase, la préposition introduit un complément de nom, un complément de verbe et un complément circonstanciel, qui n'est pas obligatoire. Dans le cadre de complément de nom, la préposition de indique :

1. un complément déterminatif (la capitale de la Belgique, la peur de mourir, chacun de nous, le livre de Pierre, une barre de chocolat, un homme de génie);

2. une apposition (la ville de Paris, faire ce geste de chasser une mouche);

3. un complément de l'adjectif (certain du succès, soucieux de tomber malade, fier de ses enfants).

Cependant, des compléments de ce type ne se construisent pas uniquement avec la préposition de ; on peut en avoir d'autres, comme p.ex. : le nettoyage par le vide, un mot pour rire, une histoire sans morale, une planche à repasser, ou la rose, reine des fleurs, exhale un parfum agréable, prêt à partir, semblable à milles soirées.

En se joignant au verbe, elle marque:

1. un complément d'objet indirect (douter de l'avenir, se contenter de peu, rêver d'une vie heureuse, dépendre du succès de cette entreprise, dissuader Marc de faire cette folie, se soucier de l'avenir de son fils);

2. un complément d'agent (être méprisé des honnêtes gens, être admiré de tous, être couvert de neige) ;

3. un attribut (être traité d'ignorant) ;

4. différents compléments circonstanciels (venir de France, échapper de prison, allonger une robe de 2 centimètres, mourir de faim, frotter d'huile, citer de mémoire, avancer de trois pas).

Comme dans le cas du syntagme nominal, aussi dans le contexte verbal, la préposition de n'est pas la seule qui permette de marquer toutes les fonctions énumérées plus haut. En outre, les hésitations quant à la distribution des prépositions sont nombreuses ; 
cette seconde difficulté est due, selon H. Bat-Zeev Shyldkrot et S. Kemmer (1995), à la fois à l'étape finale de la grammaticalisation (« nous considérons le cas de sélection obligatoire comme une étape dans un long processus de grammaticalisation, qui se manifeste par une restriction minimale du choix, où toutes les variations possibles ont fini par disparaître » (p. 209)) et à la thèse que les prépositions sont porteuses de sens, indépendamment de la sélection obligatoire et de la préférentialité d'usage (p.ex. commencer à / de, continuer à / de, s'efforcer à / de, s'empresser à / de).

Pour ce qui est du syntagme prépositionnel, la préposition de fait partie de ces syntagmes qui expriment entre autres :

1. le temps (de bon matin, de temps en temps, à partir de, d'habitude);

2. le lieu (près de, loin de, en avant de, de côté) ;

3. la quantité (de plus, de moins en moins, d'autant plus, de nouveau);

4. le point de vue (d'ailleurs, de mon côté, d'une part ... de l'autre).

Dans ce groupe, il y aura des prépositions et des adverbes. Toutefois, ce type de constructions ne sera pas examiné, étant donné la complexité du phénomène qui demande une étude séparée.

Le point de départ des présentes analyses repose principalement et de manière un peu perverse, car cette conception s'oppose aux principes de la linguistique cognitive, sur l'idée que les prépositions, en tant qu'opérateurs syntaxiques, sont privées de sens, lequel est contenu dans l'expression d'argument (La Sicile appartient à l'Italie) ou dans l'expression prédicative (une statue de Miron) dont elles font partie (Karolak 1984, 1993 ; Grochowski 1975, 1995, 2005). Par conséquent, le rôle des prépositions consisterait à fournir des instructions servant à interpréter les rapports entre les unités lexicales (Kleiber 1999). Ceci dit, nous admettons l'idée suivante : les prépositions sont des catégories sémantiques en ce sens qu'elles possèdent différentes valeurs devenant saillantes selon le contexte d'emploi (une statue de Miron vs une statue de Vénus) et qu'il y a un invariant sémantique, c'est-à-dire un sens de base qui se retrouve dans toutes les occurrences.

\section{LA PRÉPOSITION DE : UN POINT DE VUE COGNITIF}

H. Bat-Zeev Shyldkrot et S. Kemmer (1995) constatent que la préposition de possède à la fois un sens dynamique et statique, les deux liés à l'idée de relation intrinsèque entre le trajecteur et le landmark. Dans le cas du sens dynamique, le trajecteur émerge du landmark, en revanche le sens statique se fonde sur la relation partie-tout.

En effet, si on tient compte des origines latines de la préposition française $d e$, sa fonction était de marquer le début, la source ou l'origine d'un objet par rapport à l'autre. Principalement, la provenance impliquait une localisation spatiale et/ou temporelle; puis, par extension métaphorique, ce rapport s'est répandu sur d'autres types de rapports. Ainsi, l'emploi prototypique, p.ex. Marc est / provient / vient de Paris passe en Marc saute de joie, interprété comme causal. Toutefois, c'est la joie qui est à l'origine de l'action de sauter, alors cet emploi garderait la valeur de source (ou de provenance). 
Cette valeur est également à noter dans les emplois suivants : un fromage de chèvre, un vent d'orage, un coup de pied, la peur de mourir, certain du succès, soucieux de tomber malade, fier de ses enfants, citer de mémoire, sortir de l'école, oublier de, rêver de.

Pour dire p.ex. un fromage de chèvre, il faut tout d'abord une chèvre pour pouvoir faire du fromage. Dans le syntagme un vent d'orage, c'est l'orage qui donne naissance au vent. Il n'y aurait pas de coup de pied sans le pied qui donne un coup. Dans la peur de mourir, c'est la mort qui suscite un sentiment de peur.

Par ailleurs, les constructions [de + nom/infinitif] sont analysées à travers l'étude des traits intrinsèques à l'objet-trajecteur. Il est difficile ici de parler d'une relation entre deux objets, puisque ce deuxième objet n'est pas un landmark au sens proposé par R. Langacker $(1987,2008)$. Plusieurs linguistes français utilisent le terme de fond, c'est-à-dire de deuxième plan par rapport auquel on conceptualise l'objet-trajecteur. Selon J.-M. Fortis (2004 : 3), « les notions de trajecteur (trajector) et de site (landmark) sont absolument parallèles à celles de figure / fond mais Langacker leur attribue une portée qui excède largement le domaine spatial ». Cette remarque est pertinente si on tient compte de toutes les valeurs des prépositions, y compris donc celles qui ne sont pas nécessairement spatiales. Toutefois, il faut rester prudent, parce que dans l'imagerie langackerienne, le terme de fond est réservé pour un autre phénomène cognitif, qui se réfère à toutes les connaissances qui participent à mettre en lumière la figure, c'est-à-dire l'objet dont on parle.

Pour revenir à la fonction de la préposition de dans ce type de construction, elle consisterait plutôt à marquer l'objet ou la situation à partir desquels advient la conceptualisation. Même dans le cas d'une table de nuit, le concept de nuit sert de base pour faire émerger l'objet table de nuit, qui devient trajecteur. Cela veut dire que les fonctions des catégories de langues se constituent déjà au niveau cognitif, de conceptualisation et non seulement au niveau de langue.

Dans les constructions [adjectif + de + nom/infinitif], les adjectifs expriment un état d'âme qui résulte d'une situation ou d'un traitement de données antérieures (Remarquons que dans prêt à partir, prêt à répondre, l'adjectif prêt exprime plutôt une disposition de corps ou d'esprit (la situation est dynamique) qu'un état d'âme) (la situation est statique). Les constructions passives, comme couvert de neige, entouré d'arbres, aimé de tous, relèvent d'une conceptualisation statique, où les noms et pronoms sont de véritables landmarks, c'est-à-dire les objets qui servent à caractériser les trajecteurs. Et même si l'attention se focalise sur les trajecteurs, dans notre ligne de raisonnement, le point de départ de la conceptualisation serait les landmarks, c'est-à-dire que celui qui parle perçoit tout d'abord la neige, les arbres et les gens qui éprouvent de la sympathie envers qqn., pour ensuite décrire les objets-trajecteurs.

En ce qui concerne les constructions [verbe + de + nom/infinitif], avec certains prédicats, comme oublier et rêver, les activités qu'ils expriment ne peuvent se produire que sur la base de quelques informations précédemment traitées en discours ou en esprit. En effet, les activités d'oublier et de rêver s'appliquent à un contenu propositionnel qui est thématique.

La valeur de source apparaît aussi dans des constructions, comme chacun de nous, le livre de Pierre, citer de mémoire. En effet, dans tous ces cas, il faut avoir un point de repère, qui est landmark, pour en extraire un élément ou une action devenant saillant(e). 
Les deux premiers exemples impliquent l'idée d'appartenance à un groupe et à un individu, tandis que le dernier évoque directement l'idée de source de l'action.

Le rapport entre fille et collègue dans la fille de mon collègue se fonde sur l'idée de parenté, laquelle, à son tour, comporte l'idée de provenance ou d'origine. On pourrait se demander si le collègue de mon fils s'interpréterait exactement de la même façon et la réponse est positive, parce que le rapport de liaison entre le collègue et le fils se construit à partir du fils qui a un collègue. En d'autres termes, c'est le fils à partir duquel advient la conceptualisation d'un rapport professionnel.

Dans frotter d'huile, servir de guide, traiter de voleur, la préposition de introduit l'unité lexicale qui recourt à nos connaissances quant à l'huile, le guide et le voleur ; ces connaissances servant de point de départ dans le processus de la conceptualisation. Autrement dit, avant d'utiliser de l'huile pour frotter une surface, il faut admettre, même si cela ne doit pas être nécessairement efficace, que cette action sera propice; avant de se servir d'un objet ou d'un individu, il faut connaître leurs propriétés en rapport avec les contenus cognitifs et sémantiques des unités lexicales utilisées dans le contexte des activités en question.

Passons maintenant aux constructions verbales [verbe + de + nom/infinitif] et [verbe + de + infinitif].

Tout d'abord, il y a des verbes qui sont en relation de localité avec un argument introduit par la préposition de ; p.ex. (re)venir de France (du Mexique), provenir de Paris (par extension métaphorique on a provenir du latin), sortir de l'école (sortir d'une poche), s'échapper de prison (Kuryłowicz 1987). On note que la préposition de exprime le point de départ pour le déroulement de l'action du prédicat.

Des verbes comme s'abstenir, douter, s'emparer, parler, avertir, menacer, remercier, se souvenir, décider, se mêler s'unissent avec la préposition de pour donner accès à des syntagmes nominaux mais aussi, assez souvent, des syntagmes infinitifs (p.ex. s'abstenir de dessert / de répondre, douter de son succès, s'emparer de la station de radio, parler de tout et de rien / de politique (parler de politique et parler politique ne sont pas équivalents, selon nous, car elles relèvent de différentes conceptualisations), avertir qqn de son départ / avertir qqn de payer les taxes, menacer de sanctions, d'un revolver / d'être long, remercier de la charmante soirée (pour un bouquet de fleurs) / d'avoir fait qch, se souvenir de ce jour / d'avoir lu ce livre, décider du départ / de s'en aller, se mêler de politique municipale). Les verbes comme s'arrêter, se dépêcher entraînent la préposition de en construction infinitive (p.ex. s'arrêter de parler, se dépêcher de partir); enfin les verbes comme arrêter, accepter, admettre, conseiller, craindre, empêcher, dire, finir, interdire, permettre, pardonner, promettre, proposer, refuser, suggérer, tenter se construisent soit avec les syntagmes nominaux en fonction de COD soit avec la construction infinitive précédée de la préposition de (p.ex. arrêter la course / de courir, accepter l'invitation / de venir, conseiller du repos / de se reposer, empêcher le sommeil / de dormir, permettre la sortie / de sortir, refuser l'admission / d'admettre, tenter sa chance / de trouver).

Les prédicats de ces deux groupes sont "rhématiques », c'est-à-dire que pour être employés, ils demandent un certain fond thématique. Le rôle de la préposition de serait de marquer le point de départ de la conceptualisation. En effet, les actions de s'abstenir, d'avertir, de proposer et même de parler de qch, pour se produire, ont besoin d'une ori- 
gine qui ne doit pas être nécessairement dans le discours, mais qui peut être une situation imaginée à partir de laquelle le locuteur construit son énoncé. Comme dans le cas des verbes : se servir de qch, menacer qqn de qch - leur emploi suivi de la préposition de implique des connaissances préétablies quant à l'objet dont on se sert et les objets ou les situations qui provoquent la crainte (ou rendent une personne angoissée). Il en serait de même pour les verbes se mêler de qch et s'occuper de qch, où ce qui constitue le contenu du syntagme [de + nom] doit être connu des interlocuteurs pour que quelqu'un puisse ensuite s'en mêler ou s'en occuper.

Le problème qui se pose dans ce contexte d'analyse concerne le rôle de la motivation sémantique dans le processus de la grammaticalisation. Autrement dit, quels facteurs (conceptuels? sémantiques? autres ?) ont été décisifs au moment de choisir la préposition de dans les syntagmes, surtout infinitifs, qui expriment un argument d'objet ? Pourquoi dit-on: empêcher de faire qch et non empêcher faire qch*? Pourquoi les verbes nier et préférer, avant utilisés avec la préposition de, admettent aujourd'hui l'infinitif sans la préposition? Enfin, pourquoi aujourd'hui l'emploi de la préposition de est-il attesté avec le verbe se rappeler?

Comme le suggèrent H. Bat-Zeev Shyldkrot et S. Kemmer (1995: 216), il y a deux sources de détermination des formes, ce sont : la grammaticalisation et le contenu sémantique de la catégorie linguistique :

La grammaticalisation maximale peut donc aller de pair avec la motivation sémantique. Les processus de grammaticalisation sont d'ailleurs à la base de deux faits : d'une part, ils déterminent l'extension sémantique d'une forme donnée (...). Dans tout état synchronique, (...) on devra admettre qu'elles (les usages de la forme) ont un sens. D'autre part, la grammaticalisation entraîne une tendance générale à une perte de choix et à une obligation croissante (Lehmann 1985). Cependant, même dans les expressions où ce processus a atteint le degré extrême, où il n'existe plus aucun choix, un examen approfondi montrera un lien étroit entre les cas de sélection obligatoire et les autres.

Les chercheuses admettent aussi que

diachroniquement les variations dans l'usage des prépositions résultent des changements sémantiques qui ont eu lieu aussi bien dans les verbes en question que dans les prépositions.

Dans la ligne de notre raisonnement, d'une part, la grammaticalisation relèverait de l'évolution du contenu sémantique des catégories linguistiques et d'autre part, de la préférentialité d'emploi ; les deux facteurs déterminant la fréquence d'usage.

Si maintenant on réfléchit au schéma sémantico-cognitif de la catégorie de la préposition de en français, il aurait la forme suivante : 


\section{valeurs statiques}

fondées sur la relation / l'aspect intrinsèques

emplois : locatif, causal, d'agent inactif, attributif à base du rapport partie-tout, appartenance, partitif)

constructions : $[$ adjectif + de + nom/infinitif], $[$ nom + de + nom/infinitif], [participe passé + de + n om]

\section{invariant sémantique $=$}

point de départ de la conceptualisation de la scène perçue, réel ou préétabli

valeurs dynamiques fondées sur la relation extrinsèque (idée de pression / le lancement à l'extérieur)

emplois : complément d'objet, instrument / moyen

constructions : [verbe d'action $+\mathrm{de}+$ nom/infinitif]

\section{CONCLUSIONS}

Pour conclure la présente étude, revenons aux questions posées plus haut et à quelquesunes les complétant :

1. Quels facteurs (conceptuels ? sémantiques? autres ?) ont été décisifs au moment de choisir la préposition de dans les syntagmes, surtout infinitifs, qui expriment un argument d'objet?

2. Pourquoi dit-on: empêcher de faire qch et non empêcher faire qch*?

3. Pourquoi les verbes nier et préférer, autrefois utilisés avec la préposition de, admettent-ils aujourd'hui l'infinitif sans la préposition ?

4. Pourquoi aujourd'hui l'emploi de la préposition de est-il attesté avec le verbe se rappeler?

5. Pourquoi hésite-t-on toujours dans le cas des verbes s'efforcer $\grave{a} /$ de, commencer $\grave{a} / d e$ ?

6. Pourquoi dit-on décider de et se décider à?

Il y a différents facteurs qui déterminent l'emploi des prépositions dans les syntagmes infinitifs en fonction de complément d'objet. Ils relèvent du niveau conceptuel, linguistique (langue en tant que système, en diachronie et en synchronie) et discursif.

Au niveau conceptuel, comme on a essayé de le démontrer, la préposition de marque l'origine ou le début de la conceptualisation des situations statiques à caractère intrinsèque et dynamiques à caractère extrinsèque.

$\mathrm{Au}$ niveau linguistique, elle réalise différentes valeurs présentées plus haut, qui se négocient au cours du processus de grammaticalisation.

Au niveau discursif interviennent, entre autres, la fréquence d'usage et les préférences linguistiques d'un usager francophone concret. 
Ainsi, par exemple dans : c'est la fille de son père et c'est la fille à son papa, nous dirions que l'idée d'appartenance extrinsèque est la propriété de la préposition à (mon mec à moi, c'est à moi), tandis que la préposition de exprimerait l'idée d'appartenance intrinsèque. Il en serait de même dans le cas de l'extension métaphorique : une tasse de lait vs. une tasse à lait, où la préposition de construit l'image d'un tout et la préposition à implique par métaphore l'idée de destination (la tasse appartient = est destinée au lait). Pour : c'est ma faute et c'est de ma faute, parler politique et parler de politique, la préposition de introduit l'objet à partir duquel commence la conceptualisation. Alors, dans c'est de ma faute, la faute devient trajecteur qui exprime l'idée d'appartenance intrinsèque au landmark moi (c'est la faute commise par moi) ; dans parler de politique, c'est la politique qui devient un véritable trajecteur - thème de la conversation. Ce thème est extrinsèque à ceux qui en parlent (le landmark) en ce sens qu'il y a des événements politiques auxquels ceux qui en parlent ne s'engagent pas. Par contre, dans c'est ma faute, le trajecteur serait celui qui a commis la faute et la faute elle-même devient landmark, c'est-à-dire que celui qui se charge d'avoir commis la faute se met au premier plan de la scène conceptualisée et la faute est située au fond de la scène. Et dans parler politique, politique devient en quelque sorte le mode de communiquer et non seulement le thème de la conversation (parler selon les modalités de la politique ou politiquer). Cette question demande une recherche plus détaillée approfondie, mais comme elle s'est manifestée lors de notre étude, il nous a semblé approprié d'en faire également une présentation particulière.

Pour compléter ce premier examen de la préposition de, une étude approfondie de la préposition $a ̀$ et des constructions infinitives sans prépositions semble nécessaire. La comparaison des deux prépositions permettrait de saisir les différences d'emploi, d'établir éventuellement les préférences d'usage et d'examiner le rapport entre les prédicats mis en jeu. Elle permettrait également de prendre du recul et de réfléchir sur le rôle, peut-être surestimé dans le cadre de la linguistique cognitive, de l'invariant sémantique et de la motivation sémantique des tous les emplois des catégories de langue.

Une analyse cognitive des emplois de la préposition de en français contemporain proposée dans cet article a donc consisté à démontrer que : 1. la préposition de serait une catégorie contenant plusieurs emplois organisés autour d'emplois prototypiques ; 2. il existerait un invariant sémantique permettant de bien distinguer la valeur de la préposition de des autres qui peuvent apparaitre dans un même contexte (p.ex. une machine à écrire vs. une machine pour écrire) ; 3 . les formes simple et contractées sont motivées par la fréquence d'usage, celle-ci fondée sur la préférentialité d'emploi ; 4. le choix de la préposition se négocie simultanément aux niveaux conceptuel, linguistique et discursif.

\section{BIBLIOGRAPHIE}

Cervoni Jean, 1991, La préposition : étude sémantique et pragmatique, Paris/Louvain-la-Neuve : Duculot.

Desclés Jean-Pierre, Banyś Wiesław, 1997, Dialogue à propos des invariants du langage, Etudes Cognitives 2 : 11-36. 
ForTIS Jean-Michel, 2004, Introduction au problème de l'expression linguistique des relations spatiales et de la trajectoire, http://www.ddl.ish-lyon.cnrs.fr/trajectoire/23us23efd5ps/IntroFortis_160904. pdf.

Gilbert Éric, Guimier Claude, Krause Maxi (éds.), 2009, Actes du Colloque « Autour de la préposition », Caen : Presses Universitaires de Caen.

GiUliani Mariafrancesca, 2013, Una struttura semantica per da (con spunti per la redazione delle preposizioni nel TLIO), (in :) Diverse voci fanno dolci note. L'Opera del Vocabolario Italiano per Pietro G. Beltrami, Pär Larson, Paolo Squillacioti, Giulio Vaccaro (éds.), Alessandria : Edizioni dell'Orso, 107-117.

GrochOwski Marek, 1975, Zależności semantyczne między czasownikiem i wyrażeniem lokatywnym, Polonica 1 : 113-131.

GROCHOWSKI Marek, 1995, O możliwościach słownikowej charakterystyki semantycznej przyimkowych jednostek języka, (in :) Wyrażenia funkcyjne w systemie i tekście, Henryk Wróbel (red.), Torun : UMK, 89-98.

Grochowski Marek (red.), 2005, Przystówki i przyimki. Studia ze sktadni i semantyki języka polskiego, Torun : UMK.

KAROLAK Stanislaw, 1984, Składnia wyrażeń predykatywnych, (in :) Gramatyka wspótczesnego języka polskiego. Sktadnia, Zofia Topolińska (red.), Warszawa : PWN, 11-211.

KarolaK Stanislaw, 1993, Przyimek, (in :) Encyklopedia językoznawstwa ogólnego, Kazimierz Polański (red.), Warszawa : PWN.

KLeIBER George, 1999, Problèmes de sémantique. La polysémie en questions, Villeneuve d'As : Presses universitaires du Septentrion.

KurYŁowicz Jerzy, 1987, Studia językoznawcze. Wybór prac opublikowanych w języku polskim, Warszawa : PWN.

LAKOFF George, Johnson Marc, 1988, Metafory w naszym życiu, Warszawa : PIW.

LANGaCKer Ronald, 1987, Foundations of Cognitive Grammar, Stanford : Stanford University Press.

LANGACKer Ronald, 2003, Model dynamiczny oparty na uzusie, (in :) Akwizycja języka w świetle językoznawstwa kognitywnego, Ewa Dąbrowska, Wojciech Kubiński (red.), Kraków : Universitas, $30-114$.

LANGaCKer Ronald, 2008, Cognitive Grammar: A Basic Introduction, Stanford : Stanford University Press.

Luraghi Silvia, 2011, The Coding of Spatial Relations with Human Landmarks: From Latin to Romance, (in :) Case, Animacy, and Semantic Roles, Seppo Kittilä, Katja Västi, Jessi Ylikoski (eds.), Amsterdam/Philadelphia : Benjamins, 209-234.

PrZYBYLSKa Renata, 2002, Polisemia przyimków polskich w świetle semantyki kognitywnej, Kraków : Universitas.

ShYLdKrot Baat-Zeef Hava, Kemmer Susanne, 1995, La grammaticalisation des prépositions : concurrence et substitution, Revue Romane 2 : 206-225.

TAYLOR John,1993, Prepositions: Patterns of Polysemization and Strategies of Disambiguation, (in :) The Semantics of Prepositions: From Mental Processing to Natural Language Processing, Cornelia Zelinsky-Wibbelt (ed.), Berlin : Mouton de Gruyter, 151-175.

VANDELOISE Claude, 1993, Méthodologie et analyses de la préposition dans, Lexique 11 : 15-40.

VANDELOISE Claude, 1995, De la matière à l'espace : la préposition dans, Cahiers de grammaire 20 : $123-145$.

Zielinski-Wibbelt Cornelia (ed.), 1993, The Semantics of Prepositions: From Mental Processing to Natural Language Processing, Berlin : Mouton de Gruyter. 